\title{
Comparison and analysis of FDA reported visual outcomes of the three latest platforms for LASIK: wavefront guided Visx iDesign, topography guided WaveLight Allegro Contoura, and topography guided Nidek EC-5000 CATz
}

This article was published in the following Dove Press journal:

Clinical Ophthalmology

4 January 2017

Number of times this article has been viewed

\author{
Majid Moshirfar ${ }^{1,2}$ \\ Tirth J Shah ${ }^{3}$ \\ David Franklin Skanchy ${ }^{4}$ \\ Steven H Linn' \\ Paul Kang ${ }^{3}$ \\ Daniel S Durrie ${ }^{5}$ \\ 'HDR Research Center, Hoopes \\ Vision, Salt Lake City, UT, \\ ${ }^{2}$ Department of Ophthalmology and \\ Visual Sciences, John A Moran Eye \\ Center, University of Utah School \\ of Medicine, Salt Lake City, UT, \\ ${ }^{3}$ University of Arizona College of \\ Medicine - Phoenix, Phoenix, AZ, \\ ${ }^{4}$ McGovern Medical School, The \\ University of Texas Health Science \\ Center at Houston, TX, ${ }^{5}$ Durrie \\ Vision, Kansas City, KS, USA
}

Purpose: To compare and analyze the differences in visual outcomes between Visx iDesign Advanced WaveScan Studio ${ }^{\mathrm{TM}}$ System, Alcon Wavelight Allegro Topolyzer and Nidek EC-5000 using Final Fit ${ }^{\mathrm{TM}}$ Custom Ablation Treatment Software from the submitted summary of safety and effectiveness of the US Food and Drug Administration (FDA) data.

Methods: In this retrospective comparative study, 334 eyes from Visx iDesign, 212 eyes from Alcon Contour, and 135 eyes from Nidek CATz platforms were analyzed for primary and secondary visual outcomes. These outcomes were compared via side-by-side graphical and tabular representation of the FDA data. Statistical significance was calculated when appropriate to assess differences. A $P$-value $<0.05$ was considered statistically significant.

Results: The mean postoperative uncorrected distance visual acuity (UDVA) at 12 months was 20/19.25 $\pm 8.76,20 / 16.59 \pm 5.94$, and 20/19.17 \pm 4.46 for Visx iDesign, Alcon Contoura, and Nidek CATz, respectively. In at least $90 \%$ of treated eyes at 3 months and 12 months, all three lasers showed either no change or a gain of corrected distance visual acuity (CDVA). Mesopic contrast sensitivity at 6 months showed a clinically significant increase of $41.3 \%, 25.1 \%$, and $10.6 \%$ for eyes using Visx iDesign, Alcon Contoura, and Nidek CATz, respectively. Photopic contrast sensitivity at 6 months showed a clinically significant increase of $19.2 \%, 31.9 \%$, and $10.6 \%$ for eyes using Visx iDesign, Alcon Contoura, and Nidek CATz, respectively.

Conclusion: FDA data for the three platforms shows all three were excellent with respect to efficacy, safety, accuracy, and stability. However, there are some differences between the platforms with certain outcome measurements. Overall, patients using all three lasers showed significant improvements in primary and secondary visual outcomes after LASIK surgery.

Keywords: wavefront-guided, topography-guided, LASIK, wavefront optimized

\section{Introduction}

The prevalence of myopia nearly doubled in the last 30 years and continues to increase in the United States. ${ }^{1}$ Along with hyperopia and astigmatism, these errors of refraction are often easily corrected with lenses or surgery. With approximately 620,000 to 720,000 procedures performed annually in the last 5 years, laser-assisted in situ keratomileusis (LASIK) remains the predominant surgical procedure in the US to correct refractive error., ${ }^{2,3}$ LASIK has among the highest satisfaction rates of surgical procedures, ranging from $82 \%-98 \% .^{3}$ Yet recently there has been a growing
Correspondence: Majid Moshirfar HDR Research Center, Hoopes Vision, I 820 South State Street, Draper, Salt Lake City, UT 84020, USA

$\mathrm{Tel}+\mathrm{I} 8015680200$

Fax + I 80I 5650200

Email cornea2020@me.com 
public concern over the safety and effectiveness of LASIK procedure.

In 2013, the US Food and Drug Administration (FDA) approved its first topography guided laser. Unlike the wavefront guided lasers, which account for aberrations in the cornea, lens, and retina, topography guided lasers attempt to neutralize corneal irregularities and preserve its aspheric shape. ${ }^{4}$ Topography-guided lasers are shown to be superior for highly aberrated corneas when wavefront maps cannot be easily obtained. ${ }^{2,5}$ However, in regular corneas there is limited evidence to assert that visual outcomes of topography guided ablations are superior to wavefront guided or vice versa. $^{2}$ With the arrival of new FDA approved laser platforms, which include both wavefront-guided and topography-guided technology, these modern lasers have equipped surgeons with the ability to make more precise and accurate corrections. However, it may be important to understand the differences in these devices in order to maximize surgical outcome.

To our knowledge, there is no study comparing and analyzing the submitted FDA data for the three latest FDA approved platforms for LASIK surgery. The aim of this study is to compare and evaluate visual and refractive outcomes following surgery for astigmatism and myopia correction using iDesign Advanced WaveScan Wavefront guided technology (Abbott Medical Optics Manufacturing, Milpitas, CA, USA), Allegro Topolyzer topography system (Alcon Research, Ltd, Fort Worth, TX, USA), and Nidek topography system (Nidek Co. Ltd, Gamagori, Japan).

\section{Methods}

This retrospective study analyzes the submitted FDA summary of safety and effectiveness data from the three latest approved LASIK platforms: Star S4 IR ${ }^{\mathrm{TM}}$ Excimer Laser with iDesign Advanced WaveScan Studio ${ }^{\text {TM }}$ System (P930016/S044), ${ }^{6}$ Allegretto Wave Eye-Q Laser System with Allegro Topolyzer topography system and T-CAT treatment planning software (P020050/S12), ${ }^{7}$ and Nidek EC-5000 Excimer Laser System with topography-assisted LASIK treatment using the Final Fit ${ }^{\mathrm{TM}}$ custom ablation treatment planning software (P970053/S011). ${ }^{8}$ In this study, the devices will be referred to as Visx iDesign, Alcon Contoura, and Nidek CATz, respectively.

The study analyzed and compared primary, stratified, and secondary visual outcomes between each group. There were five primary outcomes measured in this study: 1) cumulative postoperative uncorrected distal visual acuity (UDVA; efficacy); 2) postoperative UDVA compared to preoperative corrected distal visual acuity (CDVA; efficacy); 3 ) change in lines of CDVA from preoperative measurements to postoperative (safety); 4) refractive stability over time (stability); and 5) postoperative mean spherical equivalent (MSE; accuracy). As indicated, each primary outcome represented the efficacy, safety, stability, and accuracy of the excimer lasers.

The three platforms show significant variance for preoperative spherical equivalent (Table 1). Visx iDesign incorporated eyes with a higher range of spherical equivalent and cylinder compared to the other two platforms (Table 2). Therefore, the preoperative spherical equivalent and cylinder were stratified to compare the three platforms. The stratified data were assessed for percent of eyes achieving 20/20 or better and percent of eyes within 0.5 diopters (D) of emmetropia postoperatively. Each stratified category was only analyzed if the sample size was greater than ten eyes. Such data were only available postoperatively at 6 months for Visx iDesign and 3 months for Alcon Contoura and Nidek CATz. Thus, these two postoperative intervals were used for comparison. FDA data did not include stratifications based on spherical equivalents for Nidek CATz. Therefore, it was excluded from the particular analysis.

There were three secondary outcomes analyzed in this study: 1) treatment for astigmatism correction, 2) mesopic and photopic contrast sensitivity, and 3) higher order aberrations (HOA). Astigmatism correction was evaluated by determining the correction ratio (CR) from the surgically induced refraction correction (SIRC) and intended refraction correction (IRC) by the following formula:

$$
\frac{\mathrm{SIRC}}{\mathrm{IRC}}=\mathrm{CR}
$$

Table I Patient demographics

\begin{tabular}{lllll}
\hline Surgery plan & Visx iDesign & Alcon Contoura & Nidek CATz & P-value $^{\#}$ \\
\hline Eyes $(\mathrm{n})$ & 334 & 249 & 135 & \\
Gender, male/female $(\mathrm{n})$ & $93 / 77$ & $93 / 119$ & $64 / 84$ & 0.057 \\
Age, mean \pm SD (years) & $32.3 \pm 8.31$ & $34.0 \pm 9.3$ & $35.1 \pm 8.5$ & $<0.016$ \\
Preoperative MSE (D) & $-6.21 \pm 2.78$ & $-4.61 \pm 2.43$ & $-3.57 \pm 1.45$ & $<0.0001$ \\
\hline
\end{tabular}

Note: ${ }^{\#}$-value calculated using one-way ANOVA at each time point.

Abbreviations: ANOVA, analysis of variance; MSE, mean spherical equivalent; D, diopter; SD, standard deviation. 
Table 2 FDA indications for use and range used in study

\begin{tabular}{|c|c|c|c|}
\hline $\begin{array}{l}\text { Refraction } \\
\text { parameter }\end{array}$ & Visx iDesign & $\begin{array}{l}\text { Alcon } \\
\text { Contoura }\end{array}$ & Nidek CATz \\
\hline \multicolumn{4}{|c|}{ Actual reported range } \\
\hline $\begin{array}{l}\text { Spherical } \\
\text { equivalent }\end{array}$ & -0.01 to $-12.0 \mathrm{D}$ & -0.01 to $-9.0 \mathrm{D}$ & Not reported \\
\hline Cylinder & 0 to $-8.0 \mathrm{D}$ & 0 to $-6.0 \mathrm{D}$ & -0.50 to $-4 \mathrm{D}$ \\
\hline Sphere & Up to $-12.0 \mathrm{D}$ & Up to $-9 \mathrm{D}$ & Up to $-6 \mathrm{D}$ \\
\hline \multicolumn{4}{|c|}{ Approved indication for use } \\
\hline $\begin{array}{l}\text { Spherical } \\
\text { equivalent }\end{array}$ & Up to $-11.0 \mathrm{D}$ & Up to $-7.0 \mathrm{D}$ & -1.0 to $-5.0 \mathrm{D}$ \\
\hline Cylinder & Up to $-5.0 \mathrm{D}$ & Up to $-3.0 \mathrm{D}$ & -0.5 to $-2.0 \mathrm{D}$ \\
\hline Sphere & Not provided & Up to $-8 \mathrm{D}$ & -1.0 to $-4.0 \mathrm{D}$ \\
\hline
\end{tabular}

Abbreviations: D, diopter; FDA, US Food and Drug Administration.

FDA data reported contrast sensitivity with significant changes and this was the basis for comparisons between groups. Greater than second order root mean square (RMS) was used as the comparison between groups and evaluated for percent change from preoperative to postoperative by the following formula:

$\frac{\text { Postoperative mean }- \text { Preoperative mean }}{\text { Preoperative mean }} * 100$

For each platform, different months were selected for reporting HOA by the FDA. HOA for Visx and Nidek CATz were represented at 6 months postoperatively while $\mathrm{HOA}$ data for Alcon Contoura was represented at 3 months.

\section{Statistical analysis}

Statistical analysis and graphical plotting was done with the help of Microsoft Excel 2015 and STATA version 14 (College Station, TX, USA). Calculations to determine statistical significance among three groups was done via one-way ANOVA with Bonferroni pairwise correction or Fisher's exact test for non-parametric data. For two sample calculations, statistically significant differences were determined using the two-tailed Student's $t$-test. A $P$-value less than 0.05 was considered statistically significant.

\section{Results \\ Efficacy}

The mean postoperative UDVA at 3 months was 20/17.94 $\pm 6.98,20 / 16.66 \pm 5.35$, and 20/18.67 \pm 4.33 for Visx iDesign, Alcon Contoura, and Nidek CATz, respectively. Mean postoperative UDVA at 12 months was 20/19.25 \pm 8.76 , $20 / 16.59 \pm 5.94$, and $20 / 19.17 \pm 4.46$ for the three platforms, respectively. The postoperative mean UDVA after Alcon Contoura at 3 months and 12 months showed a statistically significant difference from Visx iDesign and Nidek CATz ( $P=0.004$ and $P=0.0001$, respectively). The difference in mean UDVA for Visx iDesign and Nidek CATz at both time intervals was not statistically significant ( $P=0.711$ and $P=0.99$, respectively). At the end of 3 months $3 \%$ and $8 \%$ of eyes achieved a UDVA of 20/10 or better for Visx iDesign and Alcon Contoura, respectively (Figure 1A). At the end of 12 months $3 \%$ and $16 \%$ of eyes achieved a UDVA of $20 / 10$ or better, respectively (Figure 1B). These values were not reported at either interval for Nidek CATz. All three platforms had nearly $100 \%$ of eyes with $20 / 40$ or better vision at both 3 months and 12 months, greatly exceeding the FDA cutoff for efficacy (Figure 1A and B).

The total percentage of eyes that had postoperative UDVA equal to or greater than preoperative CDVA at 3 months were $69 \%, 89 \%$, and $79 \%$ for Visx iDesign, Alcon Contoura and Nidek CATz, respectively (Figure 2A). The same comparison at 12 months was $65 \%, 89 \%$, and $81 \%$ for Visx iDesign, Alcon Contoura, and Nidek CATz, respectively (Figure 2B). Nidek CATz obtained a postoperative UDVA of two lines or better than preoperative CDVA in $13 \%$ and $18 \%$ of treated eyes at both postoperative time intervals, respectively, a value larger than the other two platforms (Figure 2).

\section{Safety}

In at least $90 \%$ of treated eyes at 3 months and 12 months, all three lasers showed either no change or a gain of CDVA (Figure $3 \mathrm{~A}$ and $\mathrm{B}$ ). Nidek CATz was the only platform to have a patient lose two lines at 3 months (Figure 3A). However, the study subject had 1+ punctate epithelial keratitis along with a few meibomian gland secretions in the right eye. At 12 months, this was completely resolved. Additionally, $6 \%, 13 \%$, and $22 \%$ of eyes achieved a gain of two lines or more of CDVA at 12 months compared to preoperative CDVA for Visx iDesign, Alcon Contoura, and Nidek CATz, respectively.

\section{Postsurgical stability and accuracy}

Preoperative measurements of mean sphere were different among the three groups. Visx iDesign initially had a statistically significant higher degree of myopia $(P<0.01$; Figure 4$)$. As per the mean preoperative cylinder, Visx iDesign started with a statistically significant higher degree of astigmatism $(P<0.01$; Figure 4). However, the difference in preoperative cylinder values between Alcon Contoura and Nidek CATz was not statistically significant $(P=0.9)$. Therefore, we compared the two for any significant differences in cylinder over 

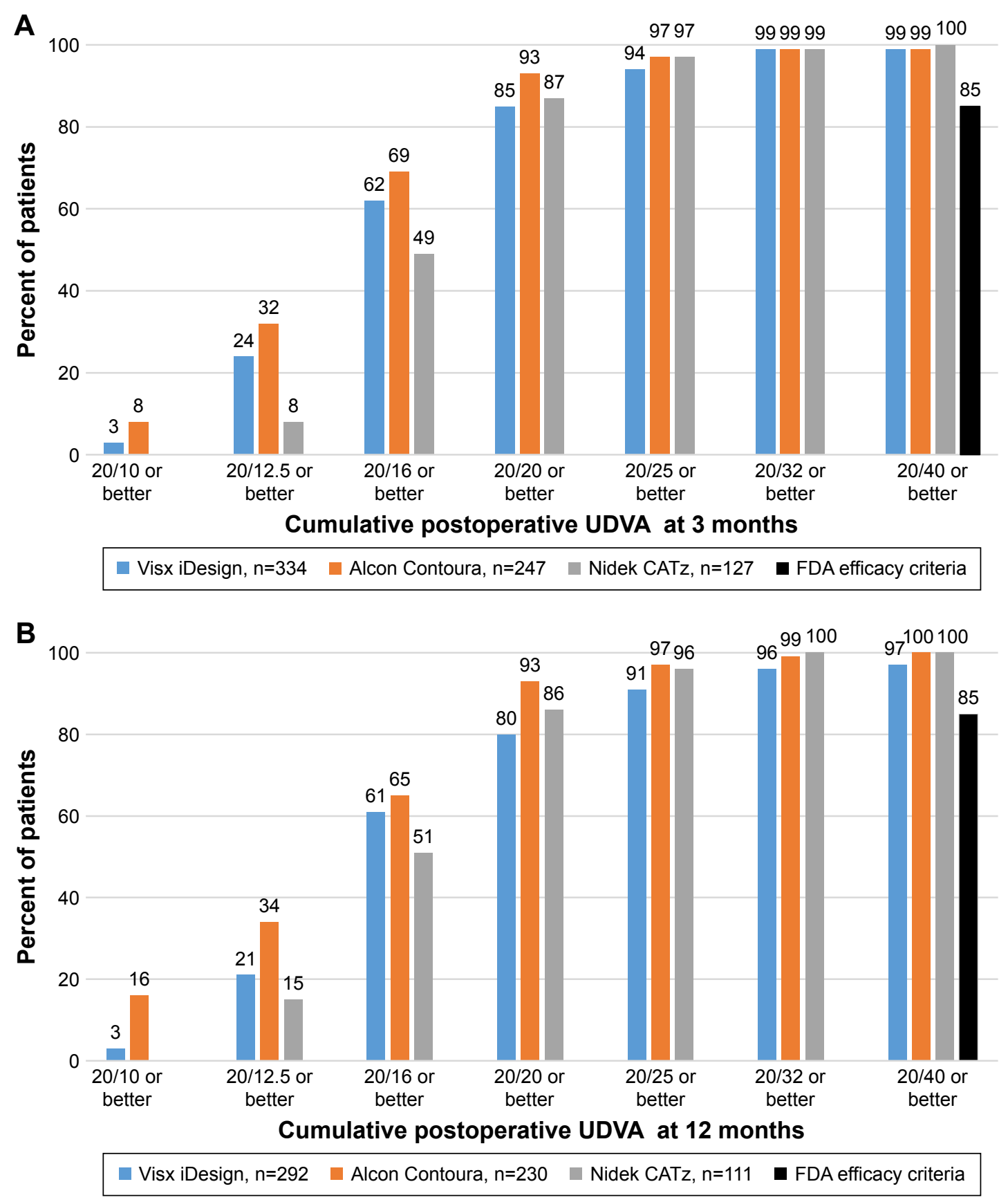

Figure I (A) The cumulative UDVA 3 months postoperatively in all three platforms. (B) The cumulative UDVA I2 months postoperatively in all three platforms. Abbreviations: UDVA, uncorrected distance visual acuity; FDA, US Food and Drug Administration.

time. At 12 months, the cylinder between the two platforms was not statistically significant $(P=1.00)$. Notably, all three platforms achieved refractive stability with time showing minimal fluctuation in spherical or cylindrical refraction between 1 month and 12 months. The same was true when evaluating MSE across time (Table 3). The sphere and cylinder of Visx iDesign were both negative causing MSE to become even more negative, whereas Alcon Contoura and Nidek CATz had a positive sphere and negative cylinder causing MSE to be closer to zero. This will be important when evaluating MSE for all three platforms.

Due to preoperative differences that were considered statistically significant, MSE could not be compared. However, it was noted that all platforms showed great refractive stability with minimal fluctuations in MSE from 1 month to 12 months.

Figure 5 shows $91 \%$ of eyes using Nidek CATz and 95\% of eyes using Alcon Contoura to have a higher proportion 


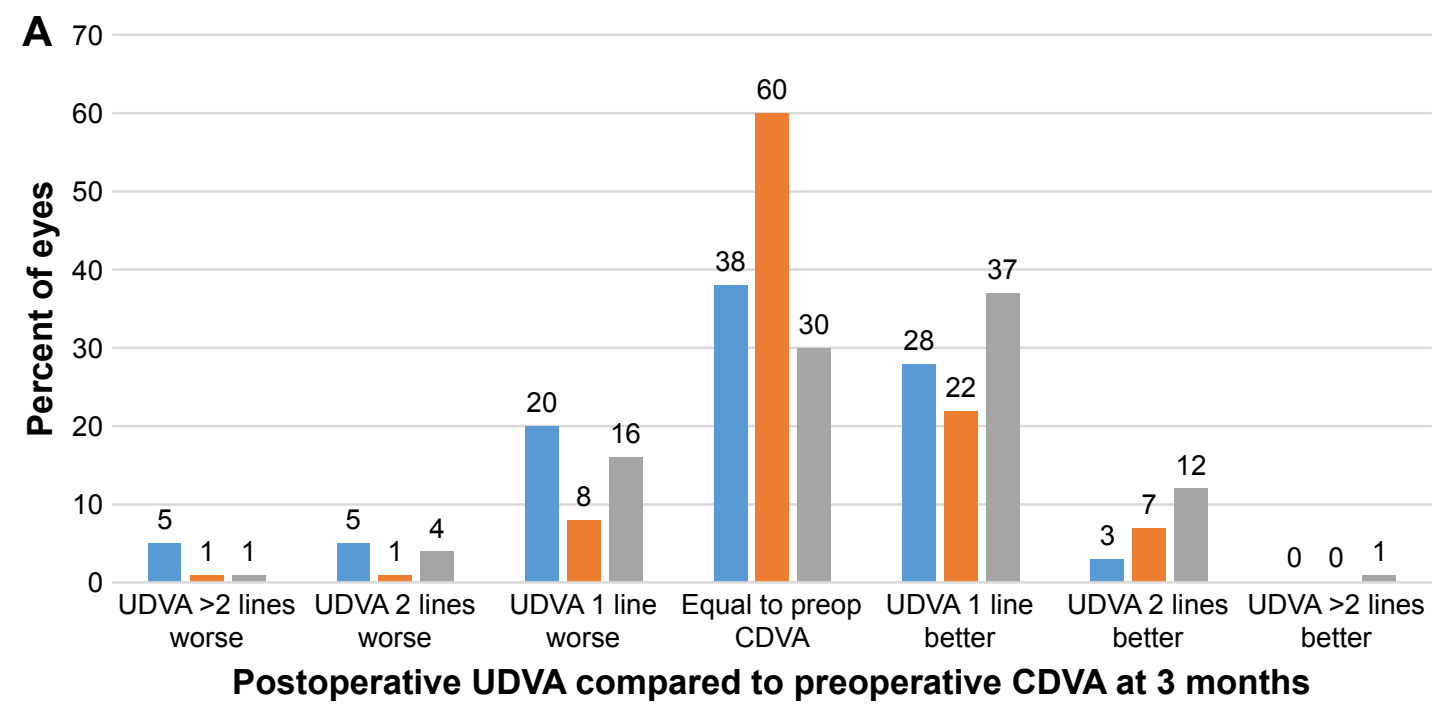

B 70

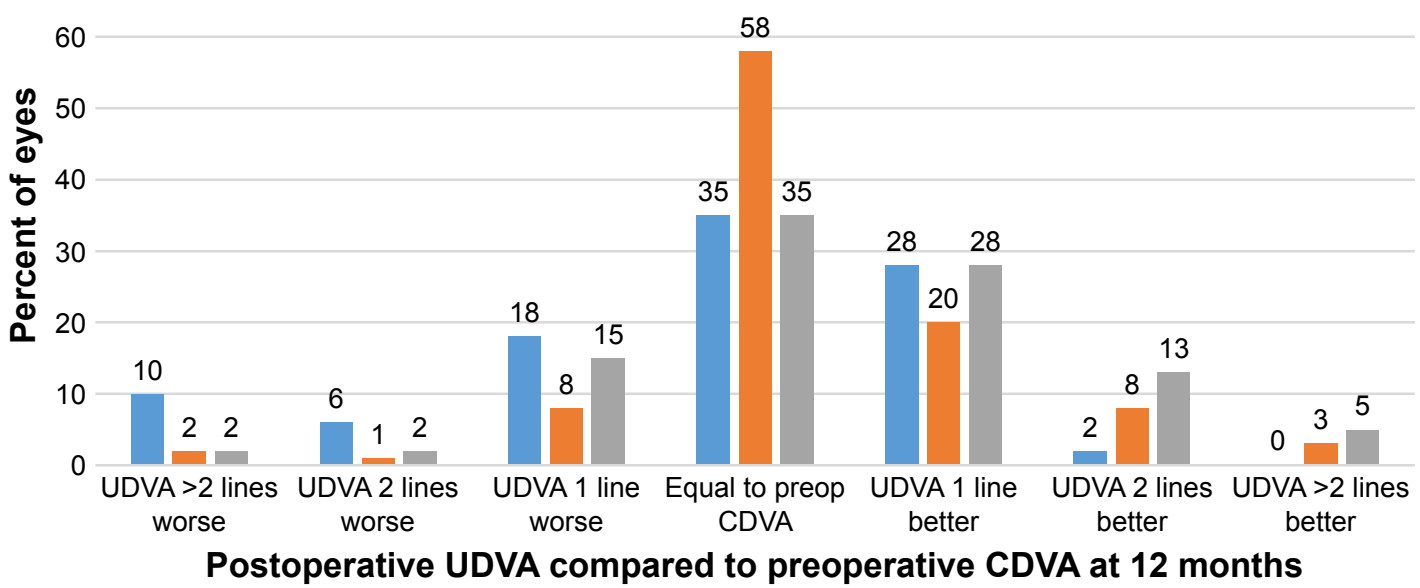

Visx iDesign $\square$ Alcon Contoura $\square$ Nidek CATz

Figure 2 (A) Postoperative UDVA compared to preoperative CDVA at 3 months for all three platforms. (B) Postoperative UDVA compared to preoperative CDVA at 12 months for all three platforms.

Abbreviations: UDVA, uncorrected distance visual acuity; CDVA, corrected distance visual acuity.

of eyes achieve within $\pm 0.5 \mathrm{D}$ of emmetropia at 6 months. At least $90 \%$ of eyes using all three platforms achieve within $\pm 1.0 \mathrm{D}$ of emmetropia at 6 months (Figure 5).

\section{Stratified preoperative MSE and cylinder}

We stratified the data into subgroups based on preoperative spherical refraction and cylinder in order to draw more accurate conclusions from similar comparisons. Comparisons for Nidek CATz based on stratified spherical equivalent could not be made due to lack of available FDA data.

At stratified ranges of -1.01 to $-2 \mathrm{D},-3.01$ to $-4 \mathrm{D}$ and -7.01 to $-8 \mathrm{D}$ for preoperative spherical equivalent, Alcon Contoura had significantly more patients $(P<0.001$ at all ranges) who achieved a 20/20 or better postoperatively
(Figure 6A). The other stratified ranges are very comparable and show no significant differences. Alcon Contoura achieved a significantly greater percentage of eyes within $0.5 \mathrm{D}$ of emmetropia at nearly all stratified cylinders than Visx iDesign $(P<0.001$ at all indicated ranges; Figure 6B).

When stratifying based on cylinder, Alcon Contoura had a significantly greater percentage of eyes with $20 / 20$ or better at lower ranges $(-0.01$ to $-1.01 \mathrm{D}$ and -1.01 to $-2 \mathrm{D}$; $P=0.029$ and $P<0.001$, respectively; Figure 7A). At all other stratified cylinder ranges, the Visx iDesign had a slightly larger percentage of eyes that achieved 20/20 or better than Alcon Contoura, although not statistically significant (Figure 7A). At nearly all ranges, Alcon Contoura had a statistically significant better outcome of eyes achieving within $0.5 \mathrm{D}$ of emmetropia 


\section{A 70}

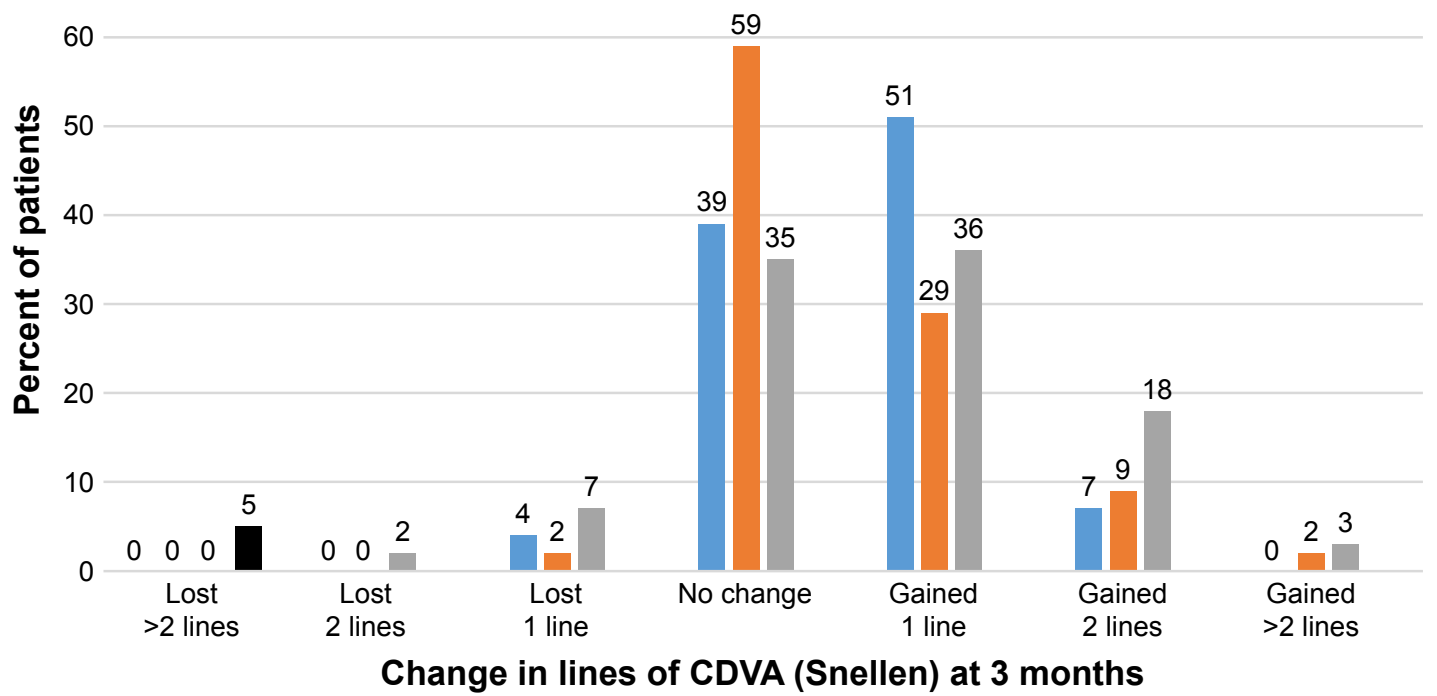

B 70

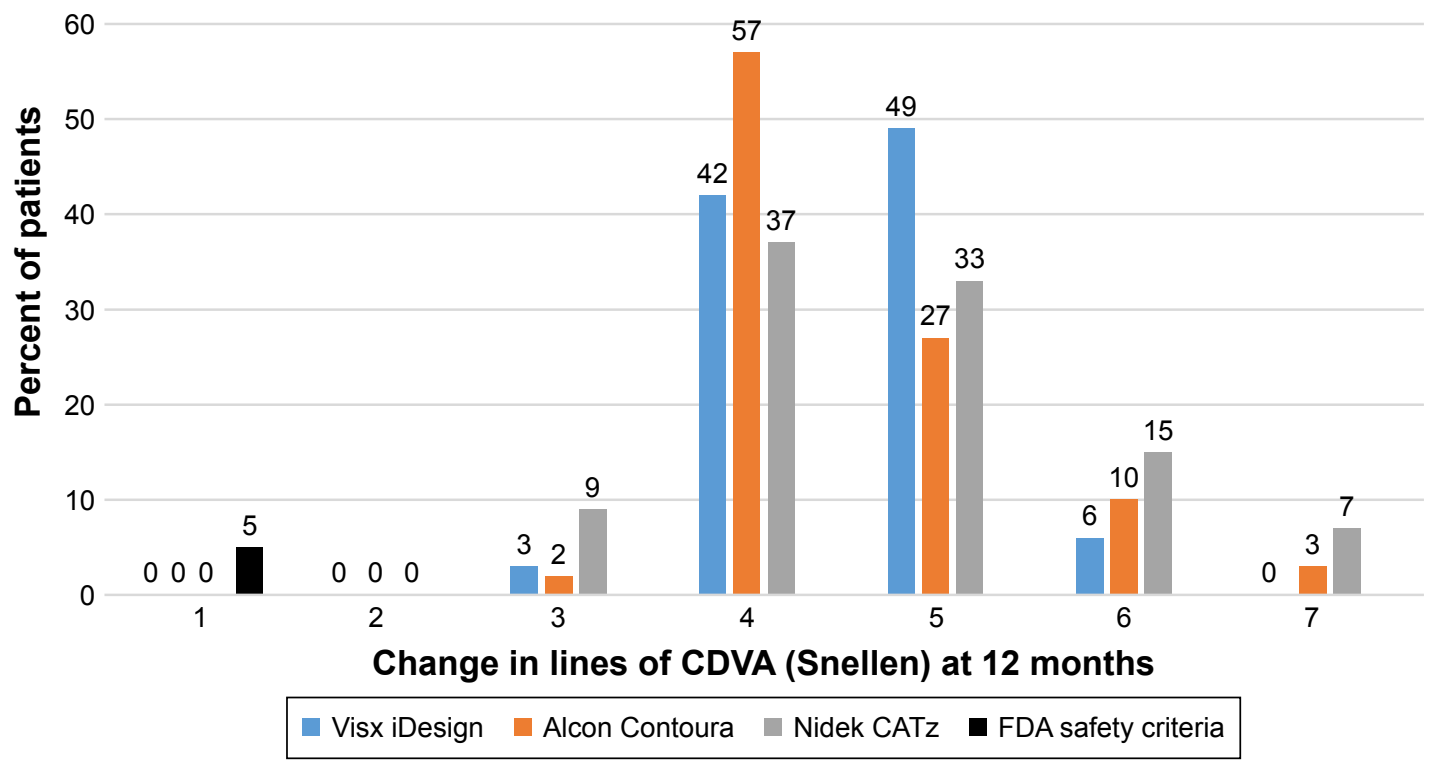

Figure 3 (A) Change in CDVA lines from preoperative to postoperative at 3 months. (B) Change in CDVA lines from preoperative to postoperative at I2 months. Abbreviations: CDVA, corrected distance visual acuity; FDA, US Food and Drug Administration.

than the other platforms (Figure 7B). Results for Nidek CATz were limited for higher cylindrical eyes $(<-2 \mathrm{D})$ due to a small sample size (less than ten eyes).

\section{Correction of astigmatism}

The CR measures the SIRC over the intended refractive correction (IRC; Table 4). The correction ratio of Alcon Contoura (1.17 and 1.02) from 0 to -1.0 was higher than the other two platforms (Figure 8). Visx iDesign did not have any reported eyes between 0 to $-0.5 \mathrm{D}$. Therefore, it was not evaluated at this stratified range. At higher stratified preoperative cylinders $(-1.0$ to $-4.0 \mathrm{D})$, the CR of the platforms shows no significant differences.

\section{Contrast sensitivity and $\mathrm{HOA}$}

Visx iDesign and Alcon Contoura had the greatest clinically significant increase postoperatively at 6 months in mesopic (41.3\%) and photopic (31.9\%) contrast sensitivity, respectively (Figure 9A and B). Nidek CATz had the smallest clinically significant decrease in both mesopic (3.8\%) and 

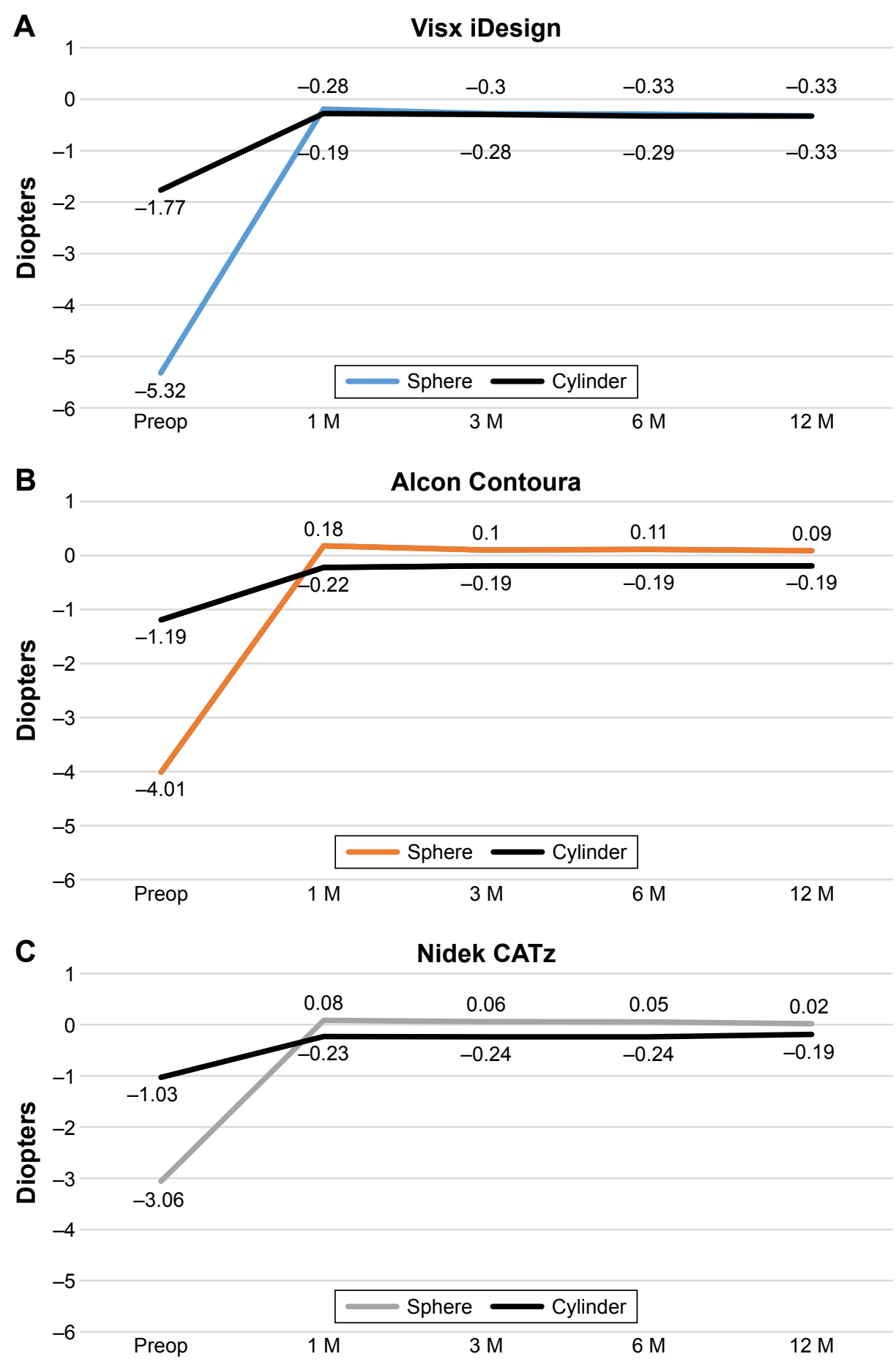

Figure 4 (A-C) Illustrations of the change of mean manifest refractive sphere (MRS) and cylinder over time for Visx iDesign, Alcon Contoura, and Nidek CATz, respectively.

Abbreviations: MRS, manifest refractive sphere; preop, preoperative; M, month.

photopic $(6.7 \%)$ contrast sensitivity and the largest percent of eyes with no change after surgery (Figure 9A and B).

When evaluating RMS of HOA, the magnitude is quite small for Alcon Contoura since it evaluated only the corneal aberrations whereas both Visx iDesign and Nidek CATz evaluated HOA of the entire optical system (Table 5).
Therefore, it was not possible to compare findings of HOA between all three lasers.

\section{Discussion}

The three platforms, Visx iDesign, Alcon Contoura, and Nidek CATz, we analyzed in this study all showed excellent 
Table 3 Changes in MSE over time

\begin{tabular}{lllll}
\hline Time period & Mean MSE \pm SD (D) & & \\
\cline { 2 - 4 } & Visx iDesign & Alcon Contoura & Nidek CATz & \\
\hline Preoperatively & $-6.21 \pm 2.78$ & $-4.61 \pm 2.43^{\mathrm{a}}$ & $-3.57 \pm 1.45^{\mathrm{b}, \mathrm{c}}$ & $<0.00 \mathrm{I}$ \\
I month & $-0.33 \pm 0.35$ & $0.06 \pm 0.36^{\mathrm{a}}$ & $-0.04 \pm\left. 0.3\right|^{\mathrm{b}, \mathrm{c}}$ & $<0.00 \mathrm{I}$ \\
3 month & $-0.43 \pm 0.39$ & $0.06 \pm 0.33^{\mathrm{a}}$ & $-0.06 \pm 0.29^{\mathrm{b}, \mathrm{c}}$ & $<0.00 \mathrm{I}$ \\
6 month & $-0.46 \pm 0.42$ & $0.01 \pm 0.35^{\mathrm{a}}$ & $-0.08 \pm 0.33^{\mathrm{b}, \mathrm{c}}$ & $<0.00 \mathrm{I}$ \\
I2 month & $-0.49 \pm 0.40$ & $0.00 \pm 0.27^{\mathrm{a}}$ & $-0.08 \pm 0.37^{\mathrm{b}, \mathrm{c}}$ & $<0.00 \mathrm{I}$ \\
\hline
\end{tabular}

Notes: ${ }^{\# P}$-value calculated using one-way ANOVA at each time point. ${ }^{2}$ Statistically significant pairwise comparison between Visx iDesign and Contoura using the two-sample $t$-test. bStatistically significant pairwise comparison between Visx iDesign and Nidek CATz using the two-sample $t$-test. 'Statistically significant pairwise comparison between Alcon Contoura and Nidek CATz using the two-sample $t$-test.

Abbreviations: D, diopter; ANOVA, analysis of variance; MSE, mean spherical equivalent; SD, standard deviation.

efficacy, safety, stability, and accuracy. They collectively had a postoperative UDVA of 20/20 or better in at least more than $80 \%$ of the eyes at 3 months and 12 months postoperatively. Eyes treated with Alcon Contoura had a mean postoperative UDVA that was significantly better than the other two platforms. In regards to refractive stability over time, Alcon Contoura and Nidek CATz both showed minimal fluctuations in sphere, cylinder, and spherical equivalent from 1 month to 12 months. Visx iDesign also showed great refractive stability and minimal fluctuation postoperatively, and even though it started at a statistically higher value of sphere, cylinder, and MSE preoperatively, all refractive mean values were within $0.5 \mathrm{D}$ of emmetropia at the end of 12 months.
No patient using any of the three platforms had a loss of two or more lines of CDVA at the end of the 12 months. Other studies evaluating Visx iDesign and Nidek CATz have confirmed the safety profile of these two platforms. ${ }^{9,10}$ They found in neither Visx iDesign nor Nidek CATz a loss of more than 1 line of CDVA. Similarly, Cummings et al ${ }^{11}$ reported that early clinical outcomes using Alcon Contoura showed no eyes lost CDVA of more than one line at 6 months. Interestingly, when comparing these platforms, we found that the demographics of the patients chosen in the Alcon Contoura FDA trials had significantly lower sphere, cylinder and spherical equivalent than those used by Visx iDesign. This may have affected the results by skewing them 120

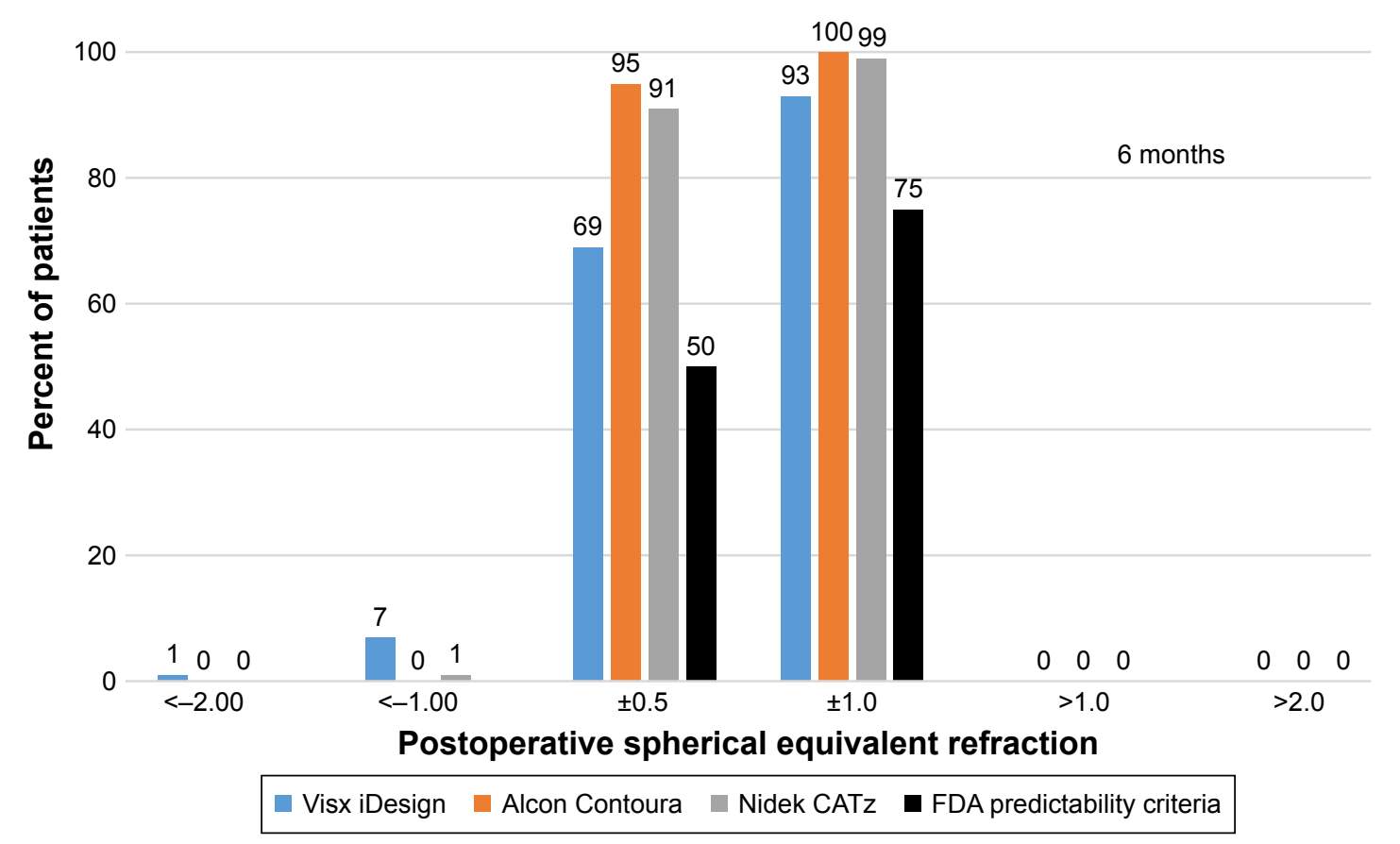

Figure 5 Percent of eyes achieving a range of postoperative spherical equivalent refraction at 6 months for all three platforms. Abbreviation: FDA, US Food and Drug Administration. 

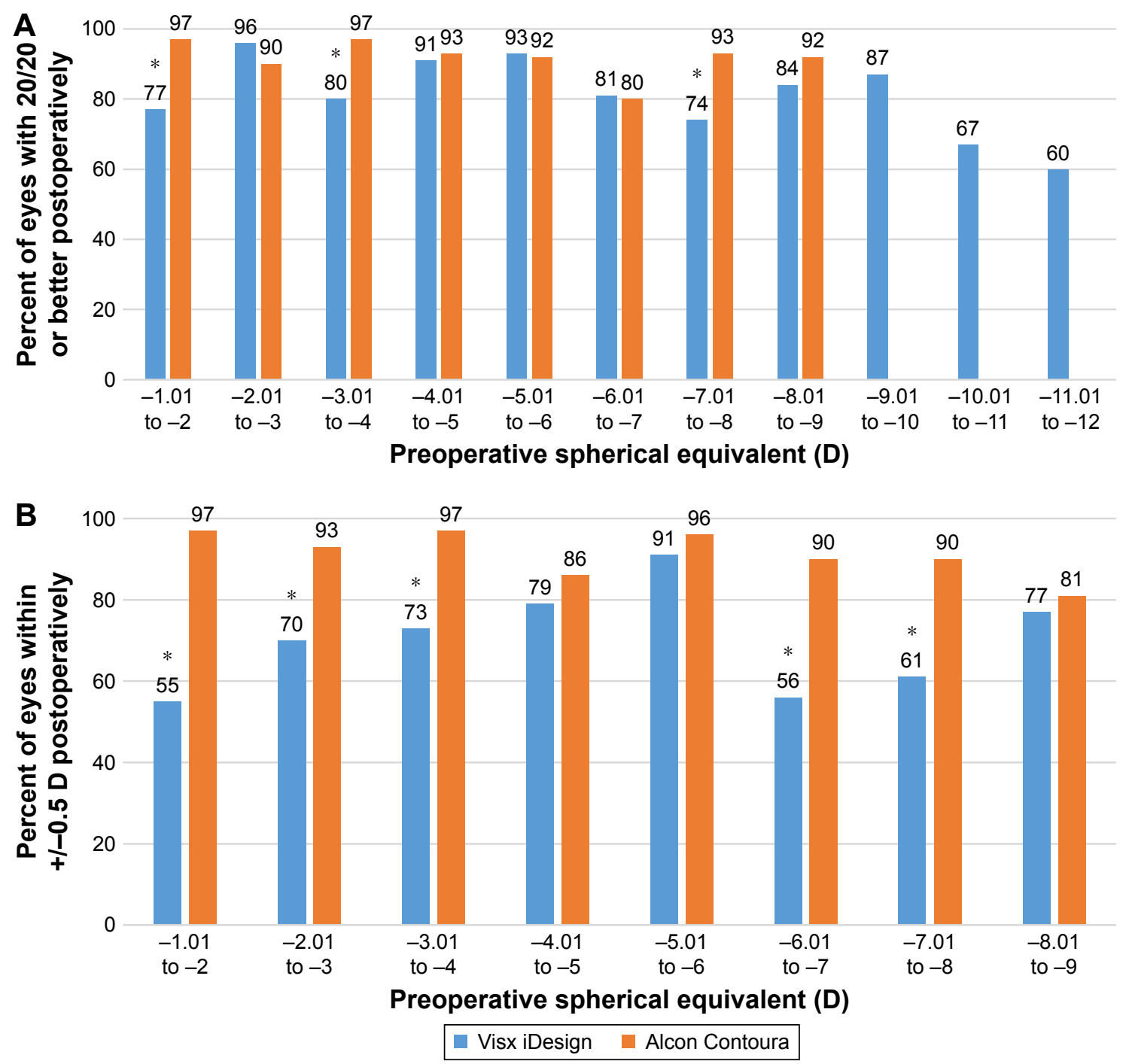

Figure 6 (A) Measuring percent of eyes achieving UDVA of 20/20 or better by stratified preoperative spherical equivalent. (B) Measuring percent of eyes within \pm 0.5 D of emmetropia by stratified preoperative spherical equivalent. In both graphs, data for Alcon Contoura and Nidek CATz were taken at 3 months and data for Visx iDesign was taken at 6 months postoperatively. Data were only included for sample size above ten. There was no FDA data stratifying Nidek CATz based on preoperative spherical equivalent, and thus, this information was excluded from the Figure. *Indicates $P$-value was less than 0.05 . Fisher's exact test was used to determine statistical significance. Abbreviations: UDVA, uncorrected distance visual acuity; D, diopter; FDA, US Food and Drug Administration.

to show greater favorability towards Alcon Contoura for visual outcomes. Visx iDesign consistently had more patients with greater preoperative sphere, cylinder, and spherical equivalent than the other two platforms. This dynamic range of refractive profiles between the platforms may have had a significant influence on the primary and secondary visual outcomes and is certainly a limitation in our analysis.

To better compare these visual outcomes, we decided to stratify preoperative spherical equivalent and cylinder. However, we were unable to compare all the visual outcome variables that we intended to due to the limitation of available FDA data. We evaluated percent of eyes achieving UDVA better than 20/20 and MSE within 0.5 D of emmetropia. Since the FDA report did not publish data based on stratified preoperative spherical equivalent for Nidek CATz, we could not evaluate outcomes of eyes using this platform. Although Visx iDesign and Alcon Contoura had fairly similar results when stratifying data based on preoperative spherical equivalent and evaluating visual acuity of 20/20 or better, Alcon Contoura was superior in certain range groups. For instance, Alcon Contoura had a better performance in patients with higher spherical equivalents $(-7.01$ to $-8 \mathrm{D})$. This is consistent with other literature in which topography guided platforms, such as Alcon Contoura, have shown to be promising in treating highly aberrated corneas. ${ }^{12}$ Nidek CATz achieved excellent results in treating eyes with low astigmatism (less than 2 diopters). Higher cylinder ranges for Nidek $\mathrm{CATz}$ could not be evaluated due to a small sample size (less than 
A

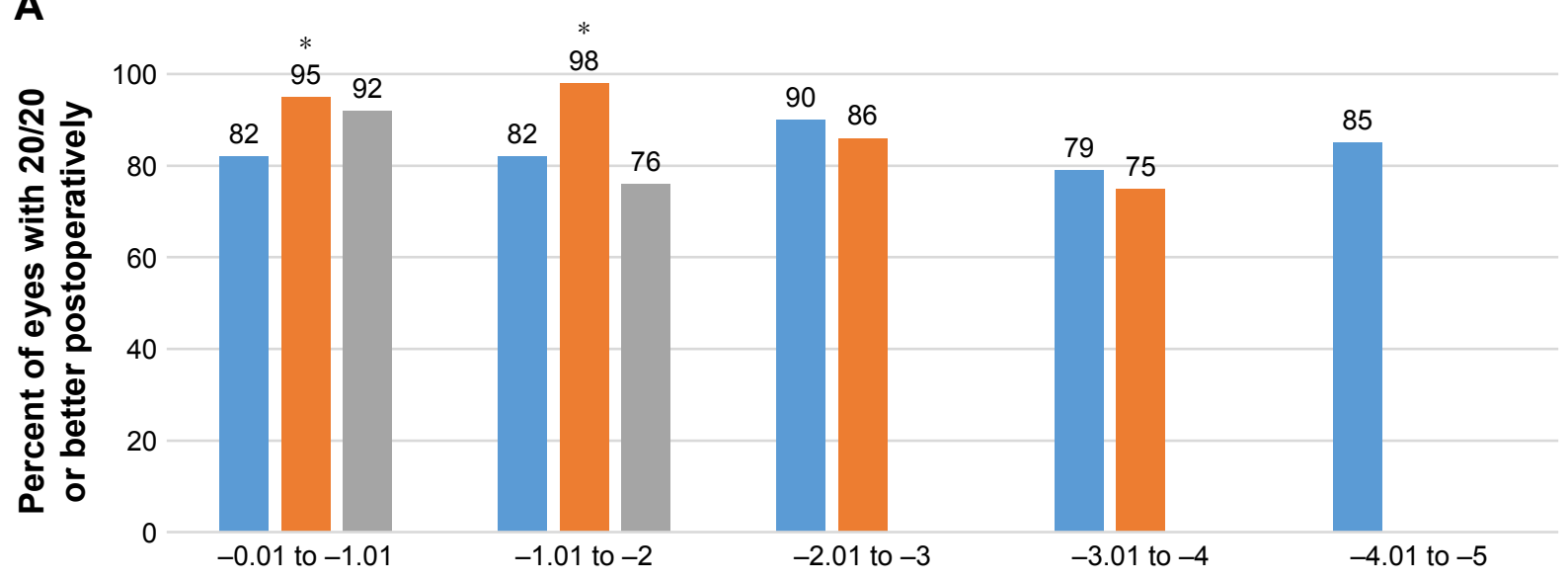

Stratified preoperative cylinder (D)

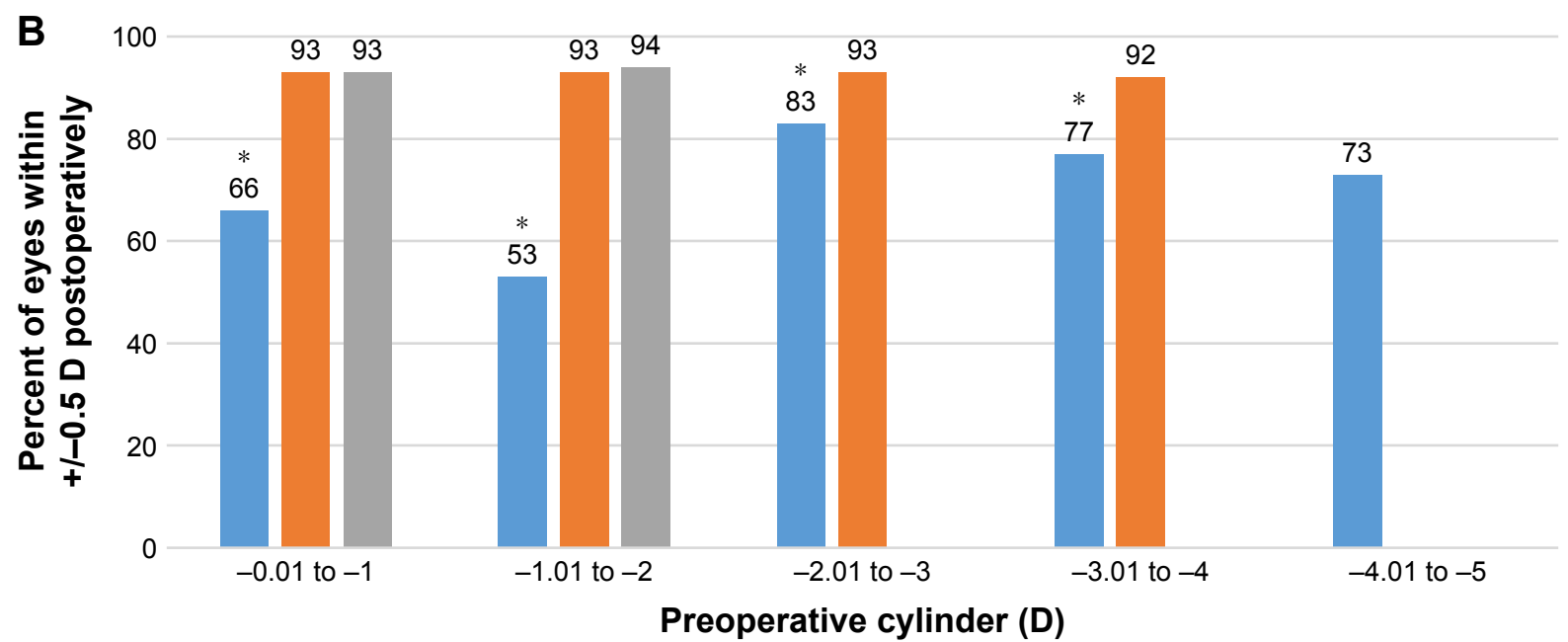

Visx iDesign alcon Contoura Nidek CATz

Figure 7 (A) Measuring percent of eyes achieving UDVA of 20/20 or better by stratified preoperative cylinder. (B) Measuring percent of eyes within \pm 0.5 D of emmetropia by stratified preoperative cylinder. In both graphs, data for Alcon Contoura and Nidek CATz were taken at 3 months and data for Visx iDesign was taken at 6 months postoperatively. Data were only included for sample size above ten. *Indicates $P$-value was less than 0.05 . Fisher's exact test was used to determine statistical significance. Abbreviations: UDVA, uncorrected distance visual acuity; D, diopter.

Table 4 Refractive correction parameters stratified by preoperative cylinder

\begin{tabular}{|c|c|c|c|c|c|c|}
\hline \multirow{3}{*}{$\begin{array}{l}\text { Preoperative } \\
\text { cylinder (D) }\end{array}$} & \multicolumn{6}{|c|}{ Mean cylinder \pm SD (D) } \\
\hline & \multicolumn{2}{|c|}{ Visx iDesign } & \multicolumn{2}{|c|}{ Alcon Contoura } & \multicolumn{2}{|c|}{ Nidek CATz } \\
\hline & IRC & SIRC & IRC & SIRC & IRC & SIRC \\
\hline-0.01 to -0.5 & \pm & \pm & $0.35 \pm 0.12$ & $0.40 \pm 0.21$ & $0.46 \pm 0.57$ & $0.45 \pm 0.19$ \\
\hline-0.51 to -1.0 & $0.86 \pm 0.13$ & $0.8 I \pm 0.25$ & $0.73 \pm 0.10$ & $0.74 \pm 0.18$ & $0.80 \pm 0.12$ & $0.76 \pm 0.19$ \\
\hline-1.01 to -2.0 & $1.61 \pm 0.31$ & $1.57 \pm 0.39$ & $1.45 \pm 0.30$ & $1.35 \pm 0.38$ & $1.38 \pm 0.24$ & $1.30 \pm 0.32$ \\
\hline-2.01 to -3.0 & $2.54 \pm 0.26$ & $2.3 I \pm 0.38$ & $2.30 \pm 0.27$ & $2.15 \pm 0.43$ & \pm & \pm \\
\hline-3.01 to -4.0 & $3.57 \pm 0.29$ & $3.29 \pm 0.49$ & $3.27 \pm 0.25$ & $3.13 \pm 0.58$ & \pm & \pm \\
\hline-4.01 to -5.0 & $4.47 \pm 0.26$ & $3.96 \pm 0.45$ & \pm & \pm & \pm & \pm \\
\hline-5.01 to -6 & $5.55 \pm 0.31$ & $5.06 \pm 0.75$ & \pm & \pm & \pm & \pm \\
\hline All & $2.5 I \pm I .58$ & $2.32 \pm 1.49$ & $0.95 \pm 0.57$ & $0.89 \pm 0.54$ & $1.27 \pm 1.09$ & $1.23 \pm 1.06$ \\
\hline
\end{tabular}

Notes: \pm denotes values that did not meet sample size threshold ( $>10$ eyes) or were not reported. Visx iDesign: postoperative 6 months. Alcon Contoura and Nidek CATz: postoperative 3 months.

Abbreviations: D, diopter; ANOVA, analysis of variance; SD, standard deviation; SIRC, surgically induced refraction correction; IRC, intended refraction correction. 


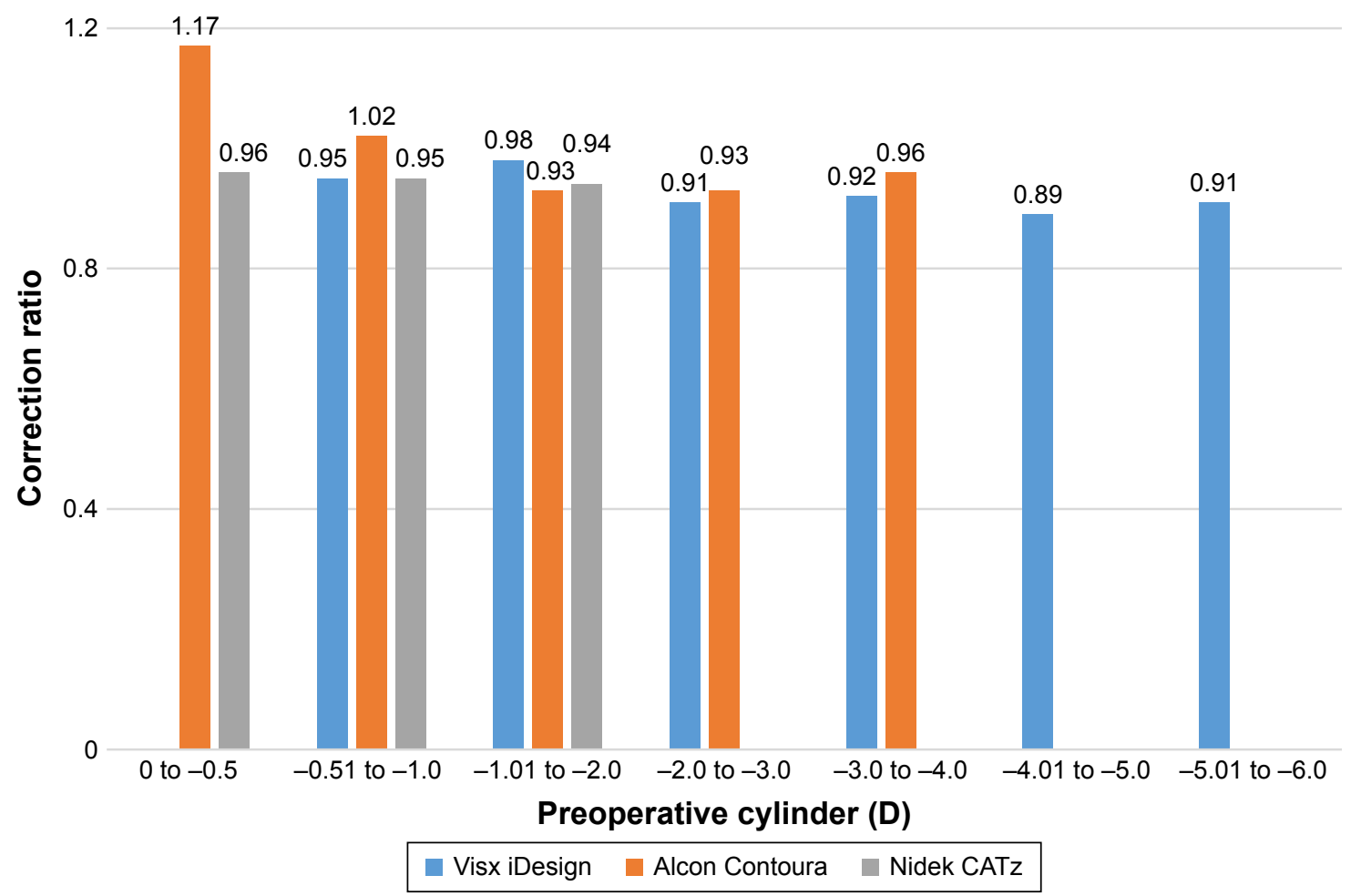

Figure $8 \mathrm{CR}$ of the three platforms stratified by preoperative cylinder magnitude. The CR is defined by SIRC/IRC. The measurements were taken at 6 months for Visx iDesign and 3 months for Alcon Contoura and Nidek CATz.

Abbreviations: $C R$, correction ratio; SIRC, surgically induced refraction correction; IRC, intended refraction correction; D, diopter.

ten eyes). It may seem at first that Visx iDesign did not perform as well as Alcon Contoura in regards to the percent of eyes achieving within $0.5 \mathrm{D}$ of emmetropia. However, Visx iDesign tended to under-correct both sphere and cylinder, thus producing a negative spherical equivalent. During the FDA study, none of the investigation sites were allowed to compensate for this slight amount of under-correction (approximately $-4 \mathrm{D}$ ). This may explain why spherical equivalent was not consistently within $0.5 \mathrm{D}$ of emmetropia and may not be a fair assessment when evaluating the two platforms. Since the approval of the Visx iDesign platform, the issue of under-correction has been reconciled with refinement of the surgical nomogram. With the implementation of the new surgeon's factor into the existing nomogram, there has been improvement in UDVA post-FDA market approval.

When stratifying preoperative cylinders and measuring CR for astigmatism, Alcon Contoura showed a higher CR than the other two platforms in patients with preoperative cylinders between 0 and $-1 \mathrm{D}$. However, the mean IRC within the stratified cohorts from -0.50 to $-4.0 \mathrm{D}$ was always lower for Alcon Contoura than it was for Visx iDesign, suggesting that Alcon Contoura tended to treat eyes with lower astigmatism in the FDA trial. ${ }^{7}$ Nonetheless, the three platforms performed similarly in treating preoperative cylinders above $-1 \mathrm{D}$.
All platforms were excellent at improving contrast sensitivity postoperatively. In particular, we found that Visx iDesign showed the greatest improvement in mesopic contrast sensitivity and Alcon Contoura showed the greatest improvement in photopic contrast sensitivity. Recent literature has shown that contrast sensitivity is significantly correlated with increases in surgically induced higher order aberrations. ${ }^{5,13,14}$ Thus, modern lasers that limit induction of higher order aberrations may actually improve contrast sensitivity. When we compared percent change of RMS of HOA, we found that Visx iDesign induced less HOA than Nidek CATz. However, this was an unfair comparison since Nidek CATz had approximately a threefold higher preoperative HOA than Visx iDesign. Treating patients with a higher baseline HOA may have also affected some of the other results, and thus control for this variable is needed for future studies.

Although Alcon Contoura showed superior outcomes for some visual results, the use of its technology can be cumbersome for novice users and initially more time-consuming for both ancillary staff and surgeon than the Visx iDesign and Nidek CATz. Cummings et a ${ }^{11}$ describes a greater degree of variability with topolyzer maps when compared to other platforms. The process usually requires capturing up to eight images and averaging the data. Often times, multiple images 

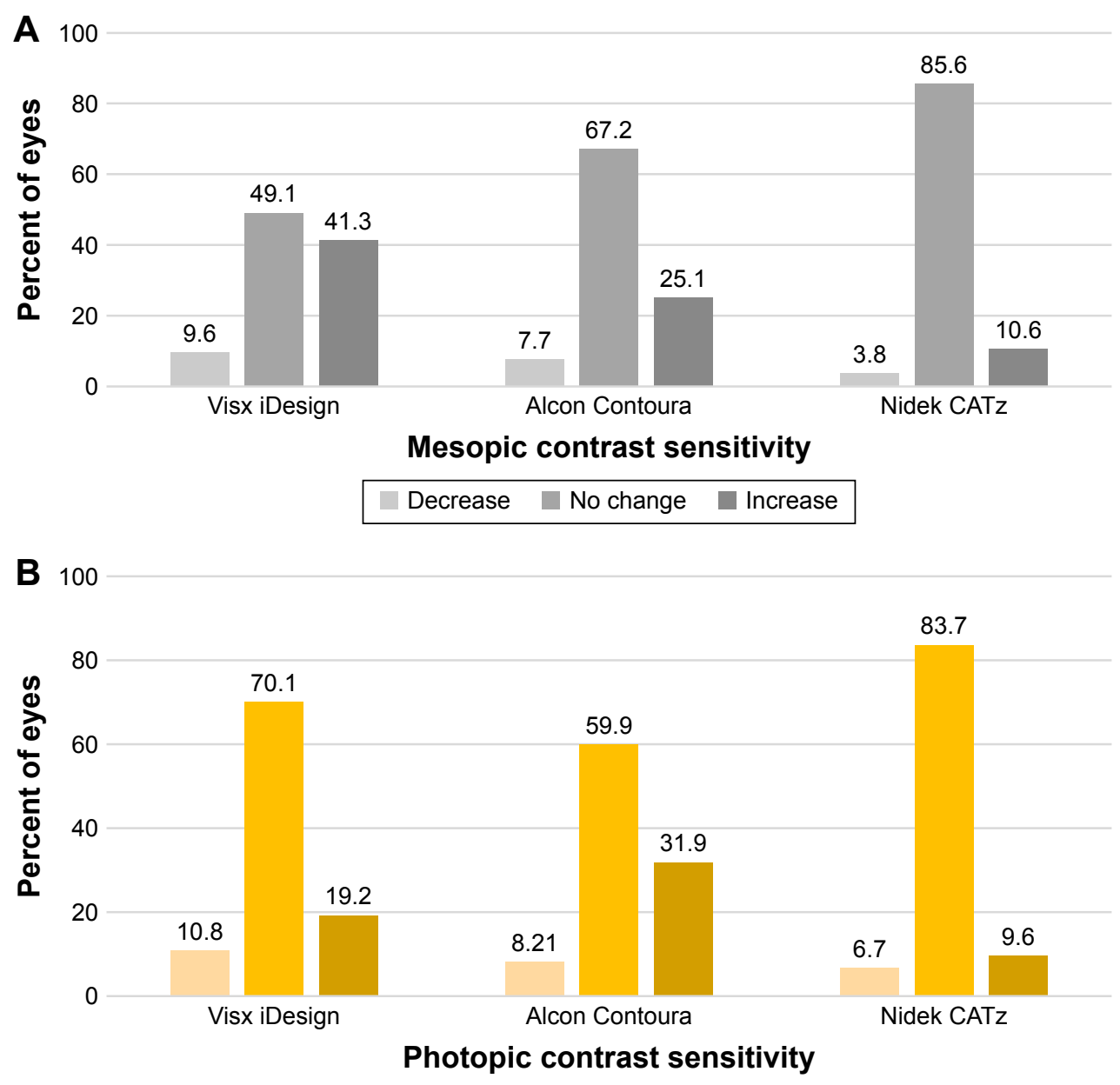

Decrease No change Increase

Figure 9 (A) Clinically significant changes from preoperative to postoperative at 6 months in mesopic contrast sensitivity among the three platforms. (B) Clinically significant changes from preoperative to postoperative at 6 months for photopic contrast sensitivity among the three platforms.

need to be repeated, and occasionally it is not even possible to perform topography because some patients, such as those with prominent brow, shadow of nose, smaller palpebral fissures, and other irregularities may not qualify. In contrast, images taken by Visx iDesign and Nidek CATz are simpler and do not require as many stringent requirements. For instance, Schallhorn et $\mathrm{al}^{9}$ describes Visx iDesign as having the capability to capture five optical measurements

Table 5 Root mean square $>$ second order

\begin{tabular}{llll}
\hline Platform & \multicolumn{2}{l}{ RMS mean $(\mu \mathrm{m}) \pm$ SD } & Change \\
\cline { 2 - 3 } & Preoperative & Postoperative (6 M) & \\
\hline Visx iDesign & $0.09 \pm 0.04$ & $0.13 \pm 0.07$ & $+44.4 \%$ \\
Alcon & $0.000537 \pm 0.000088$ & $0.000579 \pm 0.000150$ & $+7.8 \%$ \\
Contoura & & & \\
Nidek CATz & $0.243 \pm 0.101$ & $0.324 \pm 0.225$ & $+33.3 \%$ \\
\hline
\end{tabular}

Note: Measured postoperatively at 3 months and evaluated using corneal Zerninke.

Abbreviations: RMS, root mean square; SD, standard deviation; M, month. with a single capture sequence, making the process less time-consuming. Particularly, Nidek CATz uses the Optical Path Difference scan device to generate both a topographical and a wavefront snapshot of the patient's eye. Although comparatively easier to use, one drawback is that often times the treatment plan may require removal of more tissue than needed. Therefore, it requires the surgeon to make decisions based on prior experience. ${ }^{15}$

We mentioned the limitations of using different baseline data for refractive measurements of sphere, cylinder, and spherical equivalent along with HOA that may significantly impact the results for visual outcome. Other major limitations to our study include the retrospective nature of the analysis, the variable reporting methods of the FDA studies, and the way data were collected for each trial. Some outcome measurements were recorded at different months, which limited our ability to compare findings along similar timelines. This was a primary concern when evaluating the stratified 
data. Furthermore, data on specific aberrations such as trefoil and coma were either not reported in detail (in some platforms) or were acquired using different diagnostic devices and wavefront/aberrometers. By using different wavefront/ aberrometer devices for each laser, we cannot accurately compare the individual aberrations among the three platforms. In addition, pupil sizes were not given for Nidek CATz, which can also impact measurements of HOAs. We encourage future studies to compare and analyze the differences of these three platforms via a randomized, contralateral, prospective study with a large sample size to reduce some of the shortcomings we faced in our analysis. Although significantly improved from the past, we also encourage submitted FDA studies to report results in a similar format and timeline to allow for more accurate comparisons of safety and effectiveness.

We understand visual outcomes of the three platforms can be easily accessed by clinicians via the FDA website or the professional use information booklet. However, extracting FDA data between platforms can be laborious task for busy clinicians. The advantage of our report comes with the side-by-side graphical and tabular representation of the data in an unbiased format to compare and analyze differences between devices.

Overall, the three excimer lasers are exemplary of the kind of evolution we have seen in LASIK surgery. As with all surgical procedures, there will continue to be adverse events with unintended consequences. However, with advancing technology, patients using all three lasers showed significant improvements in primary and secondary visual outcomes after LASIK surgery. The primary safety and effectiveness criteria set by the FDA were not only met by all three platforms, but exceeded by a significant margin. Provided that FDA has scrutinized LASIK more than any surgery, these reports are a testament to the superior safety and effectiveness of the procedure.

\section{Acknowledgment}

This work was supported in part by an unrestricted grant from Research to Prevent Blindness, New York, NY, USA.

\section{Disclosure}

Dr Durrie is a consultant for Abbott Medical Optics. The other authors report no conflicts of interest in this work.

\section{References}

1. Vitale S, Sperduto RD, Ferri FL. Increased prevalence of myopia in the United States between 1971-1972 and 1999-2004. Arch Ophthalmol. 2009;127(12):1632-1639.

2. Mysore N, Krueger R. Advances in refractive surgery: May 2013 to June 2014. Asia Pac J Ophthalmol (Phila). 2015;4(2):112-120.

3. Bamashmus MA, Hubaish K, Alawad M, Alakhlee H. Functional outcome and patient satisfaction after laser in situ keratomileusis for correction of myopia and myopic astigmatism. Middle East Afr $J$ Ophthalmol. 2015;22(1):108.

4. Falavarjani KG, Hashemi M, Modarres M, Sanjari MS, Darvish N, Gordiz A. Topography-guided vs wavefront-optimized surface ablation for myopia using the WaveLight platform: a contralateral eye study. $J$ Refract Surg. 2011;27(1):13-17.

5. Holland S, Lin DT, Tan JC. Topography-guided laser refractive surgery. Curr Opin Ophthalmol. 2013;24(4):302-309.

6. United States Food and Drug Administration. Summary of Safety and Effectiveness Data (P930016/S044). Available at http://www.accessdata fda.gov/cdrh_docs/pdf/P930016S044B.pdf. Accessed June 1st, 2016.

7. United States Food and Drug Administration. Summary of Safety and Effectiveness Data (P020050/S12). Available at http://www.accessdata. fda.gov/cdrh_docs/pdf2/P020050S012B.pdf. Accessed June 1st, 2016.

8. United States Food and Drug Administration. Summary of Safety and Effectiveness Data (P020050/S12). Available at http://www.accessdata. fda.gov/cdrh_docs/pdf/P970053S011b.pdf. Accessed June 1st, 2016.

9. Schallhorn S, Brown M, Venter J, Teenan D, Hettinger K, Yamamoto H Early clinical outcomes of wavefront-guided myopic LASIK treatments using a new-generation Hartmann-Shack aberrometer. J Refract Surg. 2014;30(1):14-21.

10. Kermani O, Schmiedt K, Oberheide U, Gerten G. Topographic-and wavefront-guided customized ablations with the NIDEK-EC5000CXII in LASIK for myopia. J Refract Surg. 2006;22(8):754-763.

11. Cummings AB, Mascharka N. Outcomes after topography-based LASIK and LASEK with the wavelight oculyzer and topolyzer platforms. J Refract Surg. 2010;26(7):478-485.

12. Lin DT, Holland SP, Rocha KM, Krueger RR. Method for optimizing topography-guided ablation of highly aberrated eyes with the ALLEGRETTO WAVE excimer laser. J Refract Surg. 2008;24(4):S439-S445.

13. Farooqui MA, Al-Muammar AR. Topography-guided CATz versus conventional LASIK for myopia with the NIDEK EC-5000: a bilateral eye study. J Refract Surg. 2006;22(8):741-745.

14. Dougherty PJ, Waring G, Chayet A, Fischer J, Fant B, Bains HS. Topographically guided laser in situ keratomileusis for myopia using a customized aspherical treatment zone. J Cataract Refract Surg. 2008; 34(11):1862-1871.

15. Kent C. Topography-guided ablation: pros and cons. Review of Ophthalmology. 2012;20-30.
Clinical Ophthalmology

\section{Publish your work in this journal}

Clinical Ophthalmology is an international, peer-reviewed journal covering all subspecialties within ophthalmology. Key topics include: Optometry; Visual science; Pharmacology and drug therapy in eye diseases; Basic Sciences; Primary and Secondary eye care; Patient Safety and Quality of Care Improvements. This journal is indexed on

Submit your manuscript here: http://www.dovepress.com/clinical-ophthalmology-journal

\section{Dovepress}

PubMed Central and CAS, and is the official journal of The Society of Clinical Ophthalmology (SCO). The manuscript management system is completely online and includes a very quick and fair peer-review system, which is all easy to use. Visit http://www.dovepress.com/ testimonials.php to read real quotes from published authors. 